\title{
Efectos de una intervención educativa en el manejo clínico de los factores de riesgo cardiovascular en atención primaria de salud. Experiencia con Project Globe Consortium en Rusia
}

\author{
Felix Vartanyan, Honorio Silva, A.G. Avtandilov, K.N. Borisov, I.S. Shurandina, A.A. Pukhaeva, \\ Alejandro Silva, Tom Thomson, Pablo Pulido
}

Introducción. Las enfermedades cardiovasculares son la principal causa de morbimortalidad y de carga de enfermedad global. Por ello, el manejo apropiado de sus factores de riesgo (FRCV) en la prevención primaria y secundaria del daño cardiovascular es de primordial importancia. Se ha cuestionado el papel de la educación médica continuada en mejorar el desempeño profesional. Diseñamos una intervención educativa destinada tanto a médicos de atención primaria bajo el contexto de desarrollo profesional continuo como a sus pacientes, utilizando principios de educación terapéutica en un diseño abierto no controlado. El objetivo fue evaluar su impacto en parámetros cognitivos y actitudinales, y su efecto en marcadores de riesgo.

Sujetos y métodos. 30 médicos de policlínicas ambulatorias en el área metropolitana de Moscú fueron invitados a participar en un programa de actualización sobre el manejo de FRCV (72 h). Posteriormente, 600 pacientes con FRCV participaron en 12 sesiones educativas durante un periodo de tres meses. Los pacientes fueron evaluados clínicamente de forma trimestral durante 12 meses.

Resultados. Se observó una reducción significativa de los FRCV (hipertensión arterial, hipercolesterolemia, peso corporal, hiperlipidemia, tabaquismo) durante el periodo de evaluación, acompañados de cambios en el estilo de vida probablemente como reflejo de una mejor adherencia al tratamiento. La evaluación cognitiva y las actitudes hacia el control de FRCV tuvieron mejores calificaciones tanto en los médicos como en sus pacientes.

Conclusiones. La educación secuencial de médicos y de sus pacientes en el manejo de FRCV parece tener un impacto favorable en la evolución clínica de sus marcadores, pero se precisan estudios controlados para confirmar estos hallazgos preliminares.

Palabras clave. Adherencia. Atención primaria. Desarrollo profesional continuo. Educación terapéutica. Intervención educativa. Riesgo cardiovascular.

Effects of an educational intervention in the clinical management of cardiovascular risk factors in primary healthcare. Experiment with the Project Globe Consortium in Russia

Introduction. Cardiovascular diseases are the main cause of morbimortality and of burden of disease in both developed and developing countries. Therefore the appropriate management of cardiovascular risk factors (CVR) in primary and secondary prevention is highly relevant. The role of continuing medical education and continuing professional development on performance improvement has been questioned. An educational intervention addressed to primary care physicians as continuing professional development as well as to their patients with CVR was devised. Principles of therapeutic education were used. The objective was to evaluate the impact of both educational interventions on cognitive and attitudinal parameters of CVR, as well as the impact on their surrogate markers of disease progression.

Subjects and methods. 30 primary care physicians from outpatient clinics in the Moscow metropolitan area were invited to the educational intervention (lectures, seminars, workshops for a total of $72 \mathrm{~h}$ during one month). Afterwards 600 patients with CVR attending the same clinics received education on CVR (12 sessions over 3 months). Patients were clinically evaluated on quarterly bases during one year of follow up.

Results. A significant reduction in surrogate markers of CVR was observed (blood pressure, serum lipids, weight, smoking, etc.) among the patient cohort as a probable consequence of leveraged therapeutic adherence. Likewise, the knowledge and attitudes improved significantly both in physicians and their patients.

Conclusions. A sequential education of physicians and their patients with CVR seems to have a favorable impact upon surrogate markers of risk. Further controlled studies are needed to confirm these preliminary results.

Key words. Adherence. Cardiovascular risk. Continuing professional development. Educational intervention. Primary care. Therapeutic education.

Departamento de Salud Internacional; Academia Rusa de Educación Médica de Posgrado, RMAPO; Moscú, Rusia (F. Vartanyan, A.G. Avtandilov, K.N. Borisov, I.S. Shurandina, A.A. Pukhaev) Project Globe Consortium for Continuing Professional

Development; Caracas, Venezuela, y Nueva York, EE. UU. (H. Silva, T. Thomson, P. Pulido). Inter American Foundation for Clinical Research (A. Silva); Nueva York, EE. UU.

Correspondencia:

Dr. Honorio Silva. 708 Third Avenue 6F. New York, NY 10017, USA.

E-mail:

honorio.silva@globecpd.org

Financiación:

Pfizer Inc (Nueva York, EE. UU.) proporcionó los recursos financieros necesarios para la realización de este proyecto, y su subsidiaria, Pfizer Rusia (Moscú), proporcionó apoyo logístico durante su desarrollo.

(c) 2013 FEM 


\section{Introducción}

Las enfermedades cardiovasculares son la principal causa de mortalidad tanto en países desarrollados como en aquellos con economías emergentes [1,2]. Por ello, el manejo apropiado de los factores de riesgo en la prevención primaria y secundaria del daño cardiovascular es de primordial importancia.

Es bien conocido que la prevención y corrección de los factores de riesgo se traduce en una disminución de la mortalidad cardiovascular [3,4]. Médicos y profesionales de la salud que trabajan en centros de atención primaria tienen un papel fundamental en su manejo. Sin embargo, en la práctica real, la adherencia de los pacientes a las medidas preventivas y recomendaciones terapéuticas recomendadas es escasa [5,6]. Se ha atribuido al paciente la responsabilidad de su pobre adherencia. Sin embargo, la respuesta del paciente a intervenciones conductuales orientadas a mejorar la adherencia terapéutica depende también de las actitudes de los miembros del equipo de soporte en atención primaria [7], como médicos, enfermeras, nutricionistas, etc. No es fácil motivar al paciente para que cambie su estilo de vida. El entorno de la práctica de atención primaria (tiempo, espacio físico, recursos) limita la comunicación entre el paciente y su equipo de salud.

La falta de conocimiento del paciente sobre los factores de riesgo y su impacto en la salud ha sido resaltada en numerosas publicaciones $[8,9]$. Por otro lado, su opinión sobre el riesgo y la enfermedad está influida por la información que recibe de su médico, del nivel de interacción y comunicación con el equipo de salud y por la disposición a dar o recibir soporte emocional. La eficacia del tratamiento depende en gran medida de la confianza del paciente tanto en su equipo de salud como en la medicación recibida $[8,9]$. Desafortunadamente, muchos médicos consideran la esfera emocional del paciente como desligada de su marco de intervención y competencia. Por otra parte, se ha establecido que el cambio de conducta del paciente a través de la educación debería ser el principal objetivo terapéutico [10]. Por ello, si el objetivo es una intervención múltiple de los factores de riesgo (muchos de ellos conductuales o relacionados con el estilo de vida), el paciente debe dejar de ser un participante pasivo del proceso terapéutico y transformarse en un sujeto activo, preocupado y motivado para cambiar el estilo de vida y cumplir con el tratamiento médico farmacológico instituido. Para ello, la educación terapéutica del paciente (ETP) se considera una poderosa herramienta para mejorar la práctica de atención primaria, mejorar el nivel de conoci- miento del paciente sobre su condición e influir en la historia natural de la enfermedad $[11,12]$.

Sin embargo, a pesar de la disponibilidad de varias guías y publicaciones sobre el manejo del riesgo cardiovascular a través de programas de ETP, su efectividad en lograr cambios de conducta en el equipo de salud y el consiguiente impacto en salud no es bien conocida.

Por otro lado, hay evidencias crecientes sobre la efectividad de la educación médica continuada (EMC) y el desarrollo profesional continuo (DPC) en mejorar el conocimiento científico entre los médicos y en favorecer un cambio de actitud hacia el manejo de sus pacientes $[13,14]$. Sin embargo, pocos estudios han mostrado la efectividad de la EMC y el DPC en mejorar el desempeño profesional midiendo su impacto en marcadores de salud/enfermedad [15-17]. Diversos factores relacionados con la práctica profesional, el aprendiz y su ambiente de aprendizaje pueden tener una marcada influencia sobre los resultados de cualquier intervención educativa [18].

El Project Globe Consortium for Continuing Professional Development (PGC) constituye una agrupación sin fines de lucro (www.globecpd.org), concebida como una alianza estratégica de instituciones que trabajan en educación médica, caso de la FEPAFEM (Federación Panamericana de Asociaciones de Facultades y Escuelas de Medicina), sociedades científicas y profesionales de la salud con el objetivo de mejorar el desempeño profesional a través del DPC, particularmente en atención primaria, estableciendo un currículo nuclear al efecto. La iniciativa desarrollada por el PGC en cooperación con el Departamento de Salud Internacional de la Academia Rusa de Educación Medica de Posgrado se diseñó para evaluar los efectos de una intervención utilizando EMC para médicos, seguida de principios de ETP para los pacientes con riesgo cardiovascular (particularmente hipertensión arterial e hipercolesterolemia no controladas) en el control clínico de estas condiciones.

\section{Sujetos y métodos}

La intervención educativa se realizo en tres clínicas de atención medica primaria (policlínicas ambulatorias) ubicadas en los distritos administrativos Norte y Noroeste del Área Metropolitana de Moscú, durante el periodo comprendido entre febrero de 2009 y marzo del 2010. Veinte médicos de cada clínica fueron invitados a participar en el estudio y a seleccionar al azar 20 pacientes con riesgo cardio- 
vascular (particularmente hipertensión arterial o hipercolesterolemia no controlados) de una base de datos que incluía más de 3.000 pacientes ambulatorios con factores de riesgo conocidos. Tanto los médicos como sus pacientes proporcionaron el debido consentimiento informado. La evaluación se diseñó siguiendo la Declaración de Helsinki y fue aprobada por el Comité de Ética Institucional de la Academia Rusa de Educación Médica de Posgrado.

Los pacientes fueron incluidos en la evaluación de la intervención educativa de acuerdo con los siguientes criterios: hipertensión arterial en grado I o II no controlada, y/o niveles de colesterol elevados o hipercolesterolemia (niveles de colesterol sérico $>200 \mathrm{mg} / \mathrm{dL}$ o $>240 \mathrm{mg} / \mathrm{dL}$, respectivamente), acompañados o no de obesidad (índice de masa corporal $>29,0$ ), hábito tabáquico, sedentarismo o dislipidemia. Tras su aceptación en el estudio, los pacientes fueron evaluados clínicamente (examen físico y análisis de laboratorio) al iniciar la intervención educativa y luego, de forma trimestral, durante el periodo de seguimiento de un año.

\section{Intervención educativa \\ para médicos y sus pacientes}

Los médicos seleccionados participaron en sesiones de instrucción relacionadas con el manejo del riesgo cardiovascular (conferencias, seminarios y talleres de discusión), ofrecidas durante el mes previo a las sesiones de educación terapéutica. El tiempo total asignado a estas actividades fue de $72 \mathrm{~h}$. El contenido de estas sesiones se describe en la tabla I. El entrenamiento grupal (médico y sus pacientes) se realizó en el formato de educación terapéutica, abarcando los siguientes temas: hipertensión arterial (tres sesiones), síndrome metabólico y obesidad (tres sesiones), dislipidemias (tres sesiones), y tabaquismo y estilo de vida (dos sesiones). La educación fue conducida en grupos de 3-6 personas en forma de seminario interactivo y comunicación libre entre pacientes, médicos y otros profesionales de atención primaria (enfermeras, nutricionistas, etc.) durante un periodo de tres meses. El diseño del estudio puede verse en la figura.

Al finalizar el periodo de entrenamiento se distribuyó una encuesta entre los pacientes que incluía parámetros sobre información recibida y su utilidad, niveles de satisfacción y calidad de la enseñanza, temas preferidos y sugerencias para mejorar el programa en ocasiones futuras. Por otra parte, los médicos participantes también fueron evaluados en áreas referentes a la relación médico-paciente y su influencia en la adherencia terapéutica.
Figura. Diseño de la intervención educativa con Project Globe Consortium en Rusia.

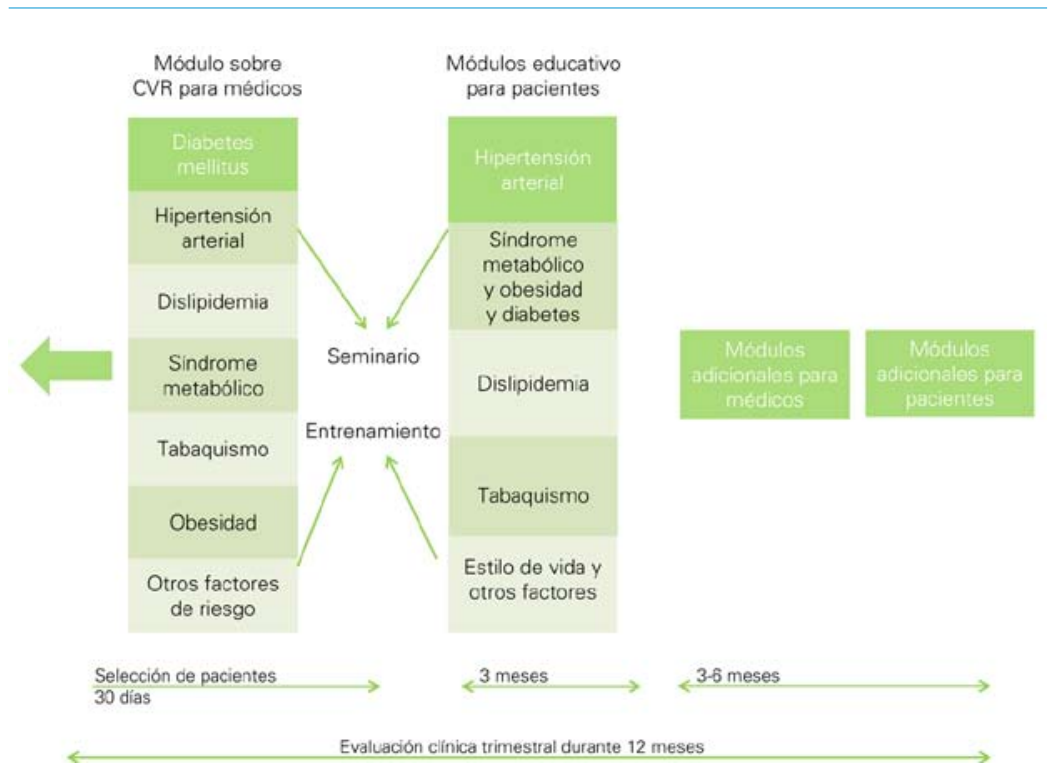

Tabla I. Syllabus del programa de educación continua sobre manejo de factores de riesgo cardiovascular.

Urgencia en la prevención primaria y secundaria de la enfermedad cardiovascular

Significado de corregir factores de riesgo

Planificación y organización de la prevención primaria y secundaria:

determinación del riesgo, estimación del riesgo, técnicas para el uso de escalas (SCORE)

Principios de manejo de factores de riesgo modificables

Hipertensión arterial, modificación del riesgo, estrategias terapéuticas

Dislipidemia, métodos de manejo bioconductual

Diabetes mellitus, prediabetes y glicemia en ayunas elevada

Síndrome metabólico y obesidad

Obesidad y salud en la mujer

Tabaquismo y estilo de vida sedentario

Alcohol y su influencia en la enfermedad cardiovascular

Prevención secundaria de enfermedad cardiovascular: farmacoterapia y cardioprotección

Aspecto psicosociales en el desarrollo de la enfermedad cardiovascular

Edad y sistema vascular: influencia de los factores de riesgo, prevención de eventos cardiacos y cerebrales

Los aspectos cognitivos, las destrezas y las actitudes en el manejo del riesgo cardiovascular fueron evaluados entre los médicos antes de la intervención y luego, de forma trimestral, durante el año de se- 
Tabla II. Factores de riesgo al iniciar el periodo de evaluación.

\begin{tabular}{lllc}
\hline & Hombres & Mujeres & Total \\
\hline Sexo & $456(76 \%)$ & $144(24 \%)$ & 600 \\
\hline Fumadores & $194(69 \%)$ & $85(31 \%)$ & $279(47 \%)$ \\
\hline Edad (mediana) & 41,7 años & 43,5 años & 42,1 años \\
\hline Prehipertensión & $30(86 \%)$ & $5(14 \%)$ & $35(6 \%)$ \\
\hline Hipertensión G1 & $229(76 \%)$ & $71(24 \%)$ & $300(50 \%)$ \\
\hline Hipertensión G2 & $131(72 \%)$ & $46(28 \%)$ & $180(30 \%)$ \\
\hline Diabetes & $22(76 \%)$ & $7(24 \%)$ & $29(5 \%)$ \\
\hline Obesidad central & $241(70 \%)$ & $83(30 \%)$ & $344(57 \%)$ \\
\hline Hábito alcohólico & $187(65 \%)$ & $102(35 \%)$ & $289(48 \%)$ \\
\hline Colesterol elevado $(>200 \mathrm{mg} / \mathrm{dL})$ & $454(72 \%)$ & $140(23 \%)$ & $594(99 \%)$ \\
\hline Hipercolesterolemia $(>240 \mathrm{mg} / \mathrm{dl})$ & $189(75 \%)$ & $65(25 \%)$ & $253(42 \%)$ \\
\hline
\end{tabular}

guimiento. Se les ofreció también material educacional (publicaciones, videos, revistas) para uso a discreción durante ese periodo. Los pacientes tuvieron oportunidad de participar de forma discrecional en sesiones relacionadas con la hipertensión arterial.

El análisis estadístico utilizo métodos descriptivos convencionales. El análisis de los cambios en los factores de riesgo se realizó a través de la prueba $t$ pareada, permitiendo un margen de error tipo A dos colas del $5 \%$.

\section{Resultados}

\section{Cambios observados en los pacientes con factores de riesgo cardiovascular}

La cohorte evaluada en este estudio incluyó 600 pacientes (144 de sexo femenino y 456 de sexo masculino; rango de edad: 30-70 años) con una alta prevalencia de factores de riesgo cardiovascular, particularmente hipertensión arterial (80\%), obesidad central (57\%), colesterol elevado (99\%) o hipercolesterolemia (42\%), habito alcohólico (48\%) o tabáquico (47\%) (Tabla II).

La intervención educativa parece haber inducido cambios significativos en la cohorte durante el periodo de evaluación (Tabla III), particularmente en el perfil de colesterol y valores de presión arterial, bien aceptados marcadores subrogados de morbilidad y mortalidad cardiovascular. Los pacientes informaron de la mejoría en la adherencia al tratamiento médico instituido. De igual modo, cambios en el estilo de vida (dieta, aumento de la actividad física) también se comunicaron a lo largo de las evaluaciones clínicas trimestrales. El cambio sostenido en los valores de presión arterial y lípidos séricos parece ser un reflejo directo del uso efectivo de medicación específica y la positiva influencia del médico tratante y de su equipo asociado.

\section{Resultados de la intervención educativa en pacientes y médicos}

Como se muestra en la tabla IV, los parámetros de la evaluación cognitiva, destrezas y actitudes de los médicos participantes en el manejo del riesgo cardiovascular mejoraron significativamente durante el periodo de observación. Una positiva actitud hacia el control del riesgo fue característica y los médicos interpretaron los resultados clínicos como una consecuencia directa del mejor conocimiento de los pacientes de su dolencia, del riesgo y su manejo apropiado.

Al finalizar el periodo de ETP se observó una disposición a realizar cambios conductuales en los pacientes incluidos en esta cohorte (Tabla V).

\section{Discusión}

Existen marcadas evidencias para prevenir o demorar la historia natural de la enfermedad cardiovascular, cáncer y diabetes a través de cambios en el estilo de vida y la dieta habitual, el cribado constante de posibles complicaciones de la enfermedad y un tratamiento farmacológico agresivo $[3,4,19]$. Ello sólo puede lograrse a través de una vigilancia epidemiológica continua, particularmente en centros de atención médica primaria donde se involucre todo el equipo de salud.

Esta fue una evaluación abierta, no controlada, de una intervención educativa para médicos de cuidados primarios en el apropiado manejo de los factores de riesgo cardiovascular, midiendo marcadores, particularmente hipertensión e hipercolesterolemia, en una cohorte de pacientes asistidos en clínicas ambulatorias en el Área Metropolitana de Moscú, en Rusia.

Los cambios en los factores de riesgo observados en la cohorte de pacientes fueron simplemente impresionantes. Al cabo de 12 meses de evaluación se 
Tabla III. Cambios en los factores de riesgo durante el periodo de evaluación (media \pm desviación estándar).

\begin{tabular}{|c|c|c|c|c|c|}
\hline & Basal & Mes 3 & Mes 6 & Mes 9 & Mes 12 \\
\hline Índice de masa corporal & $28,08 \pm 0,23$ & $\begin{array}{c}27,60 \pm 0,21 \\
t=15,29 \mathrm{a}\end{array}$ & $\begin{array}{c}27,19 \pm 0,21 \\
t=23,40^{a}\end{array}$ & $\begin{array}{c}26,86 \pm 0,21 \\
t=27,26^{a}\end{array}$ & $\begin{array}{c}26,58 \pm 0,19 \\
t=29,40^{a}\end{array}$ \\
\hline Peso (kg) & $80,14 \pm 0,66$ & $\begin{array}{c}78,76 \pm 0,63 \\
t=15,57^{\mathrm{a}}\end{array}$ & $\begin{array}{c}77,62 \pm 0,62 \\
t=23,65^{\mathrm{a}}\end{array}$ & $\begin{array}{c}76,63 \pm 0,61 \\
t=27,51^{\mathrm{a}}\end{array}$ & $\begin{array}{c}75,87 \pm 0,59 \\
t=29,78^{a}\end{array}$ \\
\hline Colesterol total (mg/dL) & $239,44 \pm 1,06$ & $\begin{array}{c}235,01 \pm 0,91 \\
t=17,20^{a}\end{array}$ & $\begin{array}{c}230,59 \pm 0,88 \\
t=28,38^{a}\end{array}$ & $\begin{array}{c}226,38 \pm 0,85 \\
t=35,73^{a}\end{array}$ & $\begin{array}{c}221,94 \pm 0,82 \\
t=41,11^{\mathrm{a}}\end{array}$ \\
\hline Colesterol-LDL (mg/dL) & $104,46 \pm 0,62$ & $\begin{array}{c}100,76 \pm 0,57 \\
t=16,72^{\mathrm{a}}\end{array}$ & $\begin{array}{c}97,05 \pm 0,56 \\
t=30,02^{\mathrm{a}}\end{array}$ & $\begin{array}{c}93,29 \pm 0,56 \\
t=41,75^{a}\end{array}$ & $\begin{array}{c}89,78 \pm 0,55 \\
t=51,02^{\mathrm{a}}\end{array}$ \\
\hline Colesterol-HDL (mg/dL) & $46,93 \pm 0,34$ & $\begin{array}{c}49,56 \pm 0,34 \\
t=-29,37 \mathrm{a}\end{array}$ & $\begin{array}{l}51,81 \pm 0,34 \\
t=-36,62^{a}\end{array}$ & $\begin{array}{l}54,25 \pm 0,36 \\
t=-38,86 a\end{array}$ & $\begin{array}{c}57,02 \pm 0,38 \\
t=-43,42{ }^{a}\end{array}$ \\
\hline Glucosa plasmática (mg/dL) & $89,76 \pm 0,91$ & $\begin{array}{c}85,92 \pm 0,78 \\
t=10,26^{a}\end{array}$ & $\begin{array}{c}83,72 \pm 0,76 \\
t=13,76^{a}\end{array}$ & $\begin{array}{c}80,96 \pm 0,79 \\
t=18,15^{a}\end{array}$ & $\begin{array}{c}77,08 \pm 0,83 \\
t=23,91^{\mathrm{a}}\end{array}$ \\
\hline Presión arterial sistólica (mmHg) & $145,80 \pm 0,74$ & $\begin{array}{c}140,90 \pm 0,60 \\
t=15,28^{\mathrm{a}}\end{array}$ & $\begin{array}{c}134,98 \pm 0,50 \\
t=24,55^{\mathrm{a}}\end{array}$ & $\begin{array}{c}130,33 \pm 0,40 \\
t=29,33^{\mathrm{a}}\end{array}$ & $\begin{array}{c}128,41 \pm 0,39 \\
t=30,87^{a}\end{array}$ \\
\hline Presión arterial diastólica $(\mathrm{mmHg})$ & $87,83 \pm 0,45$ & $\begin{array}{c}83,96 \pm 0,42 \\
t=16,30 \text { a }\end{array}$ & $\begin{array}{c}79,98 \pm 0,41 \\
t=24,45\end{array}$ & $\begin{array}{c}76,60 \pm 0,38 \\
t=29,54 \mathrm{a}\end{array}$ & $\begin{array}{c}75,42 \pm 0,35 \\
t=32,65^{a}\end{array}$ \\
\hline
\end{tabular}

a Prueba $t$ pareada en comparación con el nivel basal; todos los valores $p<0,0001$.

observó una reducción significativa en los valores de presión arterial sistólica $(-12 \%)$ y diastólica $(-14 \%)$. De igual modo, se apreciaron reducciones significativas del peso corporal $(-5,3 \%)$ y del índice de masa corporal, colesterol sérico $(-7,3 \%)$, colesterol-LDL $(-17,7 \%)$ y glucemia en ayunas $(-14,1 \%)$, acompañado de una elevación significativa en los niveles medios de colesterol-HDL (+21,5\%). Estos resultados son atribuibles a un adecuado cumplimiento de la medicación instituida y probablemente a un cambio en el estilo de vida. Más de la mitad de los pacientes incluidos comunicaron una disminución del hábito tabáquico, aunque la tasa de abandono fue sólo del $7 \%$.

Los resultados de esta experiencia fueron comparables a una experiencia similar desarrollada por el PGC en Venezuela (publicación en curso), aunque el diseño y seguimiento experimental utilizado en ese país fue algo diferente. La intervención educativa en Venezuela se realizó solamente entre médicos y utilizando tanto herramientas de educación a distancia como sesiones presenciales en educación terapéutica.

En ambos casos, el cambio de conducta observado tanto en médicos como en pacientes sería el fac- tor importante en el resultado final. La mejoría creciente en los aspectos cognitivos y actitudinales de los médicos, así como de los parámetros clínicos por ellos evaluados, es un reflejo de este fenómeno. La toma de conciencia del riesgo implícito de la condición lograda a través de la efectiva educación fue el factor primordial para inducir las modificaciones conductuales. Sin embargo, el éxito fue sólo parcial en términos de cambio en el estilo de vida, descenso de peso y hábito tabáquico, los cuales probablemente requieran una intervención más prolongada.

La diferencia fundamental con otras iniciativas fue la aplicación de principios de ETP y medición de marcadores. La ETP es parte del tratamiento no farmacológico de las enfermedades cardiovasculares, particularmente después de un evento agudo, y ayuda a los pacientes a controlar su propia enfermedad crónica. Varios estudios han mostrado la eficacia de programas de ETP en inducir cambios en el estilo de vida. Se han observado efectos favorables en relación a la morbilidad y costo-efectividad, pero no hay evidencias sobre reducción de la mortalidad. Se han estudiado diferentes tipos de intervención en ETP, pero no hay recomendaciones de estándares o métodos para proveer información 
Tabla IV. Resultados de la evaluación de conocimientos, destrezas y actitudes en el manejo del riesgo cardiovascular entre médicos participantes en el Project Globe Consortium en Rusia.

\begin{tabular}{lllll}
\hline & Basal & Mes 3 & Mes 6 & Mes 9 \\
\hline Pruebas de conocimiento (máx. 100) & $38,3 \%$ & $56,2 \%$ & $75,4 \%$ & $87,3 \%$ \\
\hline Destrezas y actitudes (máx. 100) & $42,0 \%$ & $63,4 \%$ & $84,2 \%$ & $91,5 \%$ \\
\hline
\end{tabular}

o educación, ni para la evaluación de sus resultados. Una de sus principales limitaciones es la dificultad de evaluar la adherencia a las modificaciones en el estilo de vida [12].

El diseño del estudio constituye una limitación importante para extraer mayores conclusiones, pues estos resultados sólo pueden confirmarse a través de una evaluación comparativa, incluyendo dos o tres cohortes homogéneas de pacientes y profesionales de salud que puedan ser seguidas al menos durante 12 meses. Estudios de este tipo requieren una planificación y ejecución muy rigurosas, además de los recursos financieros necesarios.

El costo y la efectividad a largo plazo de esta aproximación dual para la educación simultánea de médicos y pacientes precisan también una cuidadosa evaluación. Los cambios observados en los valores de presión arterial y lípidos deberían confirmarse a largo plazo, así como el valor potencial del reentrenamiento constante en el control del riesgo cardiovascular. Por otra parte, debido a su creciente uso y popularidad, debería compararse con otros métodos de instrucción, como la educación a distancia (e-learning). También debería considerarse la replicabilidad en otros sistemas de atención primaria diferentes al de Rusia.

\section{Bibliografía}

1. Terzic A, Waldman SA. Chronic diseases: the emerging pandemic. Clin Transl Sci 2011; 4: 225-6.

2. López AD, Mathers CD, Ezzati M, Jamison D, Murray C. Global and regional burden of disease and risk factors, 2001: systematic analysis of population health data. Lancet 2006: 367: 1747-57

3. Kovacic JC, Fuster V. From treating complex coronary artery disease to promoting cardiovascular health: therapeutic transitions and challenges 2010-2020. Clin Pharmacol Ther 2011; 90: 509-18.

4. Murray CJL, Lauer JA, Hutubessi RCW, Niessen L, Tomijima N, Rodgers A, et al. Effectiveness and costs of interventions to lower systolic blood pressure and cholesterol: a global and regional analysis on reduction of cardiovascular disease risk. Lancet 2003; 361: 717-25.

5. Kulkarni SP, Alexander KP, Lytle B, Heyss G, Peterson E. Long term adherence with cardiovascular drug regimes. Am Heart J 2006; 151: 185-91.

6. Kotseva K, Wood D, De Backer G, De Backer D, Pyorala K,
Tabla V. Cambios conductuales observados o comunicados por los pacientes $(n=600)$ al final del periodo de educación terapéutica.

\begin{tabular}{lc}
\hline Autoevaluación de los valores de presión arterial & $42,1 \%$ \\
\hline Determinación a controlar la hiperlipidemia & $57,1 \%$ \\
\hline Determinación a bajar de peso & $28,5 \%$ \\
\hline Determinación a controlar el colesterol elevado & $35,4 \%$ \\
\hline Reducción del hábito tabáquico & $53,5 \%$ \\
\hline Suspensión del hábito tabáquico & $7,3 \%$ \\
\hline Determinación a cumplir con el tratamiento instituido & $54,2 \%$ \\
\hline Asistencia a los talleres de educación terapéutica & $100,0 \%$ \\
\hline
\end{tabular}

Keil U. Cardiovascular prevention guidelines in daily practice: a comparison of EUROASPIRE I, II and III in eight European countries. Lancet 2009; 373: 929-40.

7. Pan American Health Organization. Regional strategy and plan of action on an integrated approach to the prevention and control of chronic diseases. Washington DC: PAHO; 2007.

8. Osterberg L, Blaschke T. Adherence to medication. N Engl J Med 2005; 353: 487-97.

9. Golin CE, Liu H, Hays RD, Miller LG, Ickoviscs J, Kaplan AH, et al. A prospective study of predictors of adherence to combination antiretroviral medication. J Gent Int Med 2002; 16: 269-81.

10. World Health Organization. Therapeutic patient education. Continuing education programs for health care providers in the field of prevention of chronic diseases. Copenhagen: WHO; 1998.

11. D'Ivernois JF, Albano MG. Therapeutic patient education: a new deal for medical education. Med Teach 2010; 32: 945-6.

12. Labrunee M, Pathak A, Loscos M, Coudeyre E, Casillas JM, Gremeaux V. Therapeutic education in cardiovascular diseases: state of the art and perspectives. Ann Phys Rehabil Med 2012; 55: 322-41.

13. Fahey T. Schroeder K, Ebrahim S. Interventions used to improve control of blood pressure patients with hypertension. Cochrane Database Syst Rev 2006; 4: CD005182.

14. Davis D. Does CME work? An analysis of the effect of educational activities on physician performance or health care outcomes. Int J Psychiatry Med 1998; 28: 21-39.

15. Fordis M, King JE, Ballantyne CM, Jones PH, Scheneider KH, Spann SJ, et al. Comparison of the instructional efficacy of internet based CME with live interactive CME workshops. JAMA 2005; 294: 1043-51.

16. Bloom BS. Effects of continuing medical education on improving physician clinical care and patient health: a review of systematic reviews. Int J Technol Assess Health Care 2005; 21: 380-5.

17. Mansouri M, Lockyer J. A metaanalysis for continuing medical education effectiveness. J Contin Educ Health Prof 2007; 27: 6-15.

18. Marinopoulos SS, Dorman T, Ratanawongsa N, Wilson LM, Ashar BH, Magaziner JL, et al. Effectiveness of continuing medical education. Evidence Report/Technology Assessment, no. 149. Rockville, MD: Agency for Healthcare Research and Quality; 2007.

19. Lim SS, Gaziano TA, Gakidou E, Reddy KS, Farzadfar F, Lozano R, et al. Prevention of cardiovascular disease in high risk individuals in low income and middle income countries: health effects and costs. Lancet 2007; 370: 2054-62. 